\title{
Space debris collision avoidance using a three-filter sequence
}

\author{
D. Casanova, ${ }^{1 \star}$ C. Tardioli ${ }^{2}$ and A. Lemaître ${ }^{1}$ \\ ${ }^{1}$ NaXys - Department of Mathematics, University of Namur, 8 Rempart de la Vierge, B-5000, Namur, Belgium \\ ${ }^{2}$ Department of Mechanical and Aerospace Engineering, University of Strathclyde, 75 Montrose Street, Glasgow
}

Accepted 2014 May 28. Received 2014 May 20; in original form 2014 April 14

\begin{abstract}
In the last few decades, the amount of space debris has dramatically increased, and this trend is expected to continue in the near future. Thus, there is a real risk that two objects in space orbiting about the Earth might collide. Consequently, an effective method for the detection of collisions is required in order to systematically prevent the creation of new space debris, or to study the evolution of the population of space debris after a collision occurs. This research is focused on objects orbiting in the exosphere - in low Earth orbits (LEOs) - because in the past decades these have produced the most serious damage. The methodology proposed in this paper consists of reducing the number of possible pairs of pieces of space debris into a shortlist of possible pairs at real risk of collision, using a filter sequence. This method is achieved by the following two procedures. First, an interpolation ephemerides table is built to compute the state of all the objects at several instants of time. Secondly, using the interpolation ephemerides table, the number of pairs at risk of collision is reduced by three filters. The first two filters are based on the geometry of the orbits and try to exclude pairs not undergoing orbit crossings, while the third filter searches for a time of coincidence. As a result, we have designed a powerful tool that can be used to avoid collisions between pieces of space debris.
\end{abstract}

Key words: celestial mechanics - ephemerides -Earth.

\section{INTRODUCTION}

Space debris consists of all man-made objects in orbit about the Earth that no longer serve a useful purpose. These objects can be non-active satellites, the fragments of satellites, rocket parts, the remains of explosions or collisions, etc., of all sizes and all chemical compositions.

The amount of space debris has dramatically increased in the last few decades; since Sputnik I in 1957 October, more than 7085 satellites have been launched into space for different purposes. Because of the high number of satellites orbiting the Earth, collisions between them, or explosions, can occur, either accidentally or on purpose. For example, let us mention the intentional explosion of the Fengyun $1 C$ on 2007 January 11 in a test for an antisatellite missile at an altitude of about $855 \mathrm{~km}$. The first accidental catastrophic collision occurred on 2009 February 10, at 16:56 UTC, above Siberia, between the satellites Cosmos 2251 and Iridium 33 at an altitude of about $790 \mathrm{~km}$. The destruction of these three intact satellites increased the population of space debris by about 40 percent in just $2 \mathrm{yr}$ (Pardini \& Anselmo 2011).

Currently, more than 21000 pieces of space debris larger than $10 \mathrm{~cm}$ are known to exist, grouped by the National Aeronautics and
Space Administration (NASA) in the Two Line Elements (TLE) catalogue. The estimated population of particles between 1 and $10 \mathrm{~cm}$ in diameter is approximately 500000 . The number of particles smaller than $1 \mathrm{~cm}$ exceeds 100 million, according to NASA.

The majority of the population of space debris are in low Earth orbits (LEOs). Actually, the above-mentioned catastrophic events occurred at an altitude of approximately $800 \mathrm{~km}$ above the Earth's surface. Thus, the concentration of space debris in the LEO region is a real problem for present and future space missions. However, the concentration of objects in the geostationary orbit (GEO) must also be considered as a major problem, because of the large number of satellites orbiting in that region.

The TLE catalogue contains around 21000 objects, grouping together active satellites and space debris. However, this catalogue does not consider the uncertainty of the orbits. In this paper, we consider a population of about 1000 objects whose preliminary orbits have been determined by a new orbit determination method based on the first integrals of the Kepler problem (Gronchi, Dimare $\&$ Milani 2010). The method provides the initial orbits and their uncertainty. The optical or radar observations of the population are computed via a large-scale simulation, reproducing the behaviour of a data centre for the build-up and maintenance of a complete catalogue of space debris in the upper part of the LEO region (Dimare et al. 2011). Our main goal in this paper is to provide an effective and realistic method for the detection of collisions between two objects 
of the above-mentioned population. This includes the detection of collisions between two active satellites or an active satellite and a piece of space debris, or between two pieces of space debris.

The main problem consists of computing any possible collision between each pair of objects. If we have $N \approx 21000$ objects, the number of pairs to be analysed is $N(N-1) / 2 \approx 220$ million. This means that we are dealing with a problem whose computational complexity is of the order of $N^{2}$. Thus, the main idea consists of reducing the number of pairs to be analysed using a filter sequence. Each filter will significantly reduce the number of possible pairs undergoing orbit crossing. Consequently, at the end of the filter sequence, only a shortlist of pairs will be at a real risk of collision.

The theory still largely in use was established by Hoots, Crawford \& Roehrich (1984) and it is based upon three subsequent filters. The first two filters are geometrical filters. More precisely, given a pair of objects, the first filter is based on a simple perigee-apogee computation of both objects, and the second filter is based on the minimum distance reached in space between the two paths followed by the two objects. These two filters are based on the mutual geometry of the orbits, and try to exclude pairs not undergoing orbit crossings. The third filter is the time filter, which searches for a time of coincidence when the orbit crossing occurs. In a recent work, Woodburn, Coppola \& Stoner (2010) points out the limitation in terms of efficiency resulting from the approximations of the original formulation in Hoots et al. (1984). In this paper, the previous filter approach will be significantly improved by combining geometric methods with recent mathematical and computational tools, having demonstrated their effectiveness in other contexts.

Computational complexity of order $N$ is not a problem in the context of space debris, even if $N$ is quite large. For this reason, for each of the $N$ elements of the catalogue, we compute an ephemerides table over the time-span (e.g. $10 \mathrm{~d}$ with a time-step of $36 \mathrm{~min}$ ). All the ephemerides can be stored in a similar way as the SPICE kernels used by NASA (Acton 1996). These kernels should store the ephemerides information in such a way that it can be loaded when necessary. These kernels will be stored in a binary direct access file with suitable indexing. In this way, the problem of having access to the state of any object at any instant of time computed over the time-span is solved with low computational cost. Thus, it is feasible to load thousands of these kernels into RAM memory at once.

As we mentioned before, our ephemerides table provides information about the position and velocity of space debris at fixed time-steps (time nodes). However, it is possible to compute the orbit of a piece of space debris at different times by using interpolation techniques over the data set provided by the ephemerides table. In this way, we are able to compute, for example, the position of a piece of space debris each $12 \mathrm{~min}$, reducing the time-step of the ephemerides table, or each hour, increasing the time-step with respect to the ephemerides table. These interpolations require a low computational cost. The so-called ephemerides interpolation table is then obtained. At each time node $t$ of the interpolation ephemerides table, it is possible to compute an instantaneous geocentric distance $r(t)$ and its time derivative $\mathrm{d} r(t) / \mathrm{d} t$. By using a 'regula falsi' algorithm similar to the one described by Milani et al. (2005), it is possible to compute an approximation for the time $t$, where the geocentric distance $r(t)$ reaches a maximum or minimum distance. At this point, we know the maximum and minimum distance of each object to the Earth, and consequently we can apply the first filter to exclude pairs not undergoing orbit crossings.

The second filter proposed by Hoots et al. (1984) is substantially improved by including the concept of minimum orbital intersection distance (MOID), which is commonly used in the literature about asteroids to denote the distance between two confocal elliptical trajectories. The MOID can be thought of as the minimum value reached by the two-variable function distance $d$, which represents the Keplerian distance between each pair of points in two confocal orbits. Then, given the orbits of two objects, it is possible to compute the MOID and the corresponding mutually closest points. However, the two orbits might become close at other pairs of points that correspond to the local minima of the function $d$. In the case of two elliptic, confocal and non-overlapping orbits, there are examples with up to four minimum points (Gronchi 2002). Besides, the MOID is not differentiable when it vanishes (i.e. when an orbit crossing occurs). This problem has been widely studied by Gronchi, Tommei \& Milani (2006) and Gronchi \& Tommei (2007), in which 'local orbit distances with sign', differentiable in a neighbourhood of most orbit crossings, have been introduced.

The second filter proposed here is based on the normalized MOID function. For each pair of objects, it is possible to compute their normalized MOID at any time of the ephemerides interpolation table by looking for a close approach between them. If a close approach occurs, the pair passes the second filter; otherwise, the pair is excluded from a possible collision.

The third filter, or time filter, considers the pairs of objects that pass the two previous filters. The main idea of this filter consists of computing the distance between the objects at each instant of time $t$ of the ephemerides interpolation table and checking if the distance at that time is small enough to consider the pair at risk of collision at that given time.

The only limitation of this filter sequence theory arises from space debris with poor orbit predictability, which can occur when there are unannounced manoeuvres or a large effect of drag subject to unpredictable changes in atmospheric scaleheight. In general, this is when the orbit determination becomes non-linear or nondeterministic, in which case it would be necessary to use much more complicated methods. In this paper, we compute the ephemerides of each piece of space debris with the dynamical systems developed by the naXys team, which include the Earth's gravitational potential, the luni-solar and planetary gravitational perturbations and the solar radiation pressure effect (Valk, Lemaître \& Anselmo 2008; Valk, Lemaître \& Deleflie 2009a; Valk et al. 2009b; Lemaître, Delsate \& Valk 2009; Delsate et al. 2010; Hubaux et al. 2012; Casanova \& Lemaître 2014).

The paper is organized as follows. First, we introduce the ephemerides table and the way we use it in the filter sequence. Then, we describe the three filters and we apply them to a fictitious set of 864 objects orbiting the Earth. We analyse the results obtained and, finally, we conclude and present future work.

\section{PRELIMINARIES}

In this section, we detail the number of objects to be considered hereafter, and we describe the process used to obtain the ephemerides of these objects and the way we store them by using direct access files. We also explain how to compute the ephemerides interpolation table and how to use it in the filter sequence.

\subsection{Orbit catalogue}

The TLE catalogue developed by NASA contains about 21000 objects greater than $10 \mathrm{~cm}$, grouping together active satellites and space debris. The TLE catalogue does not provide the orbit uncertainty. In this paper, we consider $N=864$ objects whose initial 
orbital conditions have been determined by a new orbit determination method, based on the first integrals of the Kepler problem (Gronchi et al. 2010), which provides the initial location of the satellites and its uncertainty. These 864 objects are in the LEO region, which means that the semimajor axis $a$ satisfies $a \leq 8600 \mathrm{~km}$.

\subsection{Structure of the ephemeris table}

The evolution of each of the $N=864$ orbits in the catalogue is computed numerically by a symplectic integration scheme (Hubaux et al. 2012), which includes in the model the Earth's gravitational potential, luni-solar and planetary gravitational perturbations and direct solar radiation pressure. In particular, we propagate each object for a time-span of $10 \mathrm{~d}$ with a time-step of $36 \mathrm{~min}$. This means that, for a given object, we have 400 time nodes represented by $t_{j}=(j-1) \times 36 \mathrm{~min}$, with $1 \leq j \leq 400$, and its corresponding state vector $\left[\boldsymbol{r}\left(t_{j}\right), \dot{\boldsymbol{r}}\left(t_{j}\right)\right]$, which represent the position and velocity vectors at each time node $t_{j}$. All these data represent the ephemerides of the given object.

On many occasions, instead of the position and velocity vectors, the ephemerides of an object are given by the classical orbital elements: the semimajor axis $a$, the eccentricity $e$, the inclination $i$, the right ascension of the ascending node $\Omega$, the argument of perigee $\omega$ and the mean anomaly $M$. However, when the eccentricity approaches zero, the argument of perigee is undefined, and when the inclination is close to zero, the right ascension of the ascending node is undefined. To overcome these problems, we use the equinoctial orbital elements, which are suitable for all eccentricities and inclinations, even for null eccentricities and inclinations. The equinoctial orbital elements (Broucke \& Cefola 1972) are given by

$$
\begin{array}{ll}
a, & p=\tan (i / 2) \sin \Omega, \\
h=e \sin (\omega+\Omega), & q=\tan (i / 2) \cos \Omega, \\
k=e \cos (\omega+\Omega), & \lambda=M+\omega+\Omega .
\end{array}
$$

The first five elements give the configuration of the orbit in space, and the last element is a fast angle variable along the trajectory.

We compute the ephemerides of the 864 objects by using the equinoctial orbital elements. The innovative part of this work is that we store the data by using direct access files with suitable indexing, in a similar way as the SPICE kernels used by NASA (Acton 1996). Direct access, also called non-sequential or random access, divides the file associated with the input/output data into fixed-length records (RECs), and allows the program to read or write data at any point in the file, directly by a record number.

For clarity, we explain the way we store the ephemerides in our research. The records are built with seven real numbers: the time and the six orbital elements. Then, given the object number one, $s=1$, the ephemerides at $t_{1}=0$ are stored in the record number $1(\mathrm{REC}=1)$, and the ephemerides at $t_{2}=36$ are stored in the record number $2(\mathrm{REC}=2)$. Analogously, the ephemerides at $t_{3}, t_{4}$, $\ldots, t_{400}$ are stored in the record numbers $3,4, \ldots, 400(\mathrm{REC}=3$, $\mathrm{REC}=4, \ldots, \mathrm{REC}=400)$. Now, the ephemerides of the object number two, $s=2$, at $t_{1}=0$ are stored in the record number 401 $(\mathrm{REC}=401)$, and so on. In general, we have an orbit index $s(1$ $\leq s \leq N=864)$ and a time index $t_{j}(1 \leq j \leq m=400)$. By using direct access methodology, the ephemerides of the orbit $s$ at time $t_{j}$, which are the orbital elements of the object $s$ at time $t_{j}$, are stored in the record number $(s-1) m+j[\operatorname{REC}=(s-1) m+j]$. In this way, the problem of having access to the ephemerides of all objects at all time nodes is solved with low computational cost.

A binary ephemerides table of 864 objects propagated for $10 \mathrm{~d}$, with a time-step of $36 \mathrm{~min}$ requires $19.4 \mathrm{Mb}$ of RAM memory.

\subsection{Interpolation of the ephemeris table}

At this point, we have stored in a direct access file, the ephemerides of the 864 objects at 400 time nodes. However, 400 time nodes might not be suitable for some particular research. In some cases, fewer than 400 time nodes are enough, but in other cases, where more precision is required, more time nodes are needed. In both cases, the ephemerides of the objects at the required time nodes are computed by using a simple linear interpolation technique.

This technique allows us to compute the interpolated ephemerides of an object at any instant of time $t$ between two time nodes $\left(t_{j}, t_{j+1}\right)$ with $1 \leq j<400$, simply by linear interpolation, because the first five equinoctial orbital elements are not angle variables and, consequently, their linear interpolation is elementary. The sixth element is an angle variable, but it does not provide any information about the geometry of the orbit, and consequently a linear interpolation for it is not necessary. Once we have the interpolated ephemerides at time $t$, we can compute the required information at that time, for example, the geocentric distance $r(t)$. This process requires the knowledge of two time nodes $\left(t_{j}, t_{j+1}\right)$ and their corresponding equinoctial orbital elements, which are stored in the direct access file (ephemerides table), and we interpolate at the time required with low computational cost and low RAM memory. Note that if we have 400 time nodes and if we interpolate once between each node, we have double the data with a low computational cost. Thus, the more we interpolate, the more data we have, but the memory required will always be the same because we only use the data stored in the ephemeris table, proving the effectiveness of the direct access files.

\section{THREE-FILTER SEQUENCE}

As mentioned in the introduction, there are about 21000 catalogued objects in the TLE catalogue, and consequently the number of pairs to be analysed is $N(N-1) / 2 \approx 220$ million. The idea of reducing significantly the number of possible pairs undergoing orbit crossing emerges from the infeasibility of considering all possible pairs.

In this paper, we consider 864 objects, which translate into 372816 possible pairs to be analysed. However, as a result of the three-filter sequence, described in this section, these pairs can be reduced into a shortlist of pairs that will be at real risk of collision.

\subsection{Filter I}

Filter I is an improvement of the perigee-apogee technique formulated by Hoots et al. (1984), whose theory considers a primary body, which is the object orbiting the Earth that must be protected, and every other object, called secondaries, that can collide with the primary. The main idea consists of a simple perigee-apogee computation of the possible pairs defined by the primary and each of the secondaries. Then, given a pair, let $q$ denote the largest of the two perigees and let $Q$ denote the smallest of the two apogees. If $q-Q$ $\geq D$, where $D$ represents a threshold distance, then the secondary does not need to be considered further because a collision cannot occur.

In a recent reassessment, Woodburn et al. (2010) have pointed out the limitation of efficiency resulting from the simple perigeeapogee computation proposed by Hoots et al. (1984). There are two main problems. First, the perigee and apogee of each pair are computed using the osculating orbital elements, which are subject to perturbations, over short and long periods. Then, if filter I uses the osculating elements, there is a need to control the perturbations and to study how they influence the computation of the perigee and 
apogee. The second problem is that the comparison of the perigee and apogee of two objects at some fixed time $t$ might lead to a false positive if one of the two either is an active satellite performing a manoeuvre or experiences a significant drag. The availability of an ephemerides table means that we can avoid using the approximation represented by the osculating elements.

Consider an object with index $s(1 \leq s \leq 864)$ in our particular catalogue. Let $E_{s}\left(t_{j}\right)$ represent the equinoctial orbital elements of the object $s$ at the time node $t_{j}$ with $1 \leq j \leq m$, where $m$ represents the number of time nodes. The values of $E_{s}\left(t_{j}\right)$ are stored in the ephemerides table. Then, it is possible to compute the equinoctial orbital elements at a generic time $t$ in the range $t_{1} \leq t \leq t_{m}$ by linear interpolation. Thus, it is possible to compute an instantaneous geocentric distance at time $t$ of the object $s$,

$r_{s}(t)=\frac{q(1+e)}{1+e \cos \varphi}$,

where $q$ is the perigee distance and $\varphi$ is the true anomaly at time $t$. The main idea of filter I is to replace the calculation of the perigee and apogee (Hoots et al. 1984) with the calculation of the absolute minimum and absolute maximum of the function $r_{s}(t)$, respectively. In order to compute the minimum and maximum of the geocentric distance at time $t$, we use the Cartesian coordinates of the position vector, $\boldsymbol{r}_{s}(t)=[x(t), y(t), z(t)] \in \mathbb{R}^{3}$. Thus, the geocentric distance and its time derivative at time $t$ are

$r_{s}(t)=\sqrt{x(t)^{2}+y(t)^{2}+z(t)^{2}}, \quad \dot{r}_{s}(t)=\frac{\boldsymbol{r}_{s}(t) \cdot \dot{\boldsymbol{r}}_{s}(t)}{r_{s}(t)}$,

where $\dot{\boldsymbol{r}}_{s}(t)=[\dot{x}(t), \dot{y}(t), \dot{z}(t)] \in \mathbb{R}^{3}$ is the Cartesian velocity vector, and (.) indicates the Euclidean scalar product in $\mathbb{R}^{3}$.

For each object, we search for the absolute minimum and absolute maximum of the function $r_{s}(t)$, for $t_{1} \leq t \leq t_{m}$, where $t_{1}$ is the initial epoch corresponding to the initial orbital conditions. For this purpose, we use a similar algorithm to the one used by Milani et al. (2005). This algorithm computes the geocentric maximum and minimum distances, using a simple 'regula falsi' algorithm between the two consecutive time nodes, where the exact $t$ that reaches the maximum and minimum is located. This problem is equivalent to finding the values where the derivative function $\dot{r}_{s}(t)$ is zero. Finally, the absolute minimum and absolute maximum of $r_{s}(t)$ can be set as

$$
\begin{aligned}
& \min \left(r_{s}\right):=\min \left\{r_{s}(t), t_{1} \leq t \leq t_{m}\right\}, \\
& \max \left(r_{s}\right):=\max \left\{r_{s}(t), t_{1} \leq t \leq t_{m}\right\} .
\end{aligned}
$$

The number of times we interpolate the function $r_{s}(t)$ depends on the number of time nodes suitable for our research, as we mention in Section 2.3.

We know the minimum and maximum distances of each object $1 \leq s \leq N=864$ to the Earth's centre, denoted by $\min \left(r_{s}\right)$ and $\max \left(r_{s}\right)$, respectively. Thus, we can compare these values for all possible pairs. Given a pair of objects $(a, b)$ with $1 \leq a<b \leq$ $N=864$, we set

$q:=\max \left\{\min \left(r_{a}\right), \min \left(r_{b}\right)\right\}$,

$Q:=\min \left\{\max \left(r_{a}\right), \max \left(r_{b}\right)\right\}$.

We say that there is no orbit crossing if the condition $|q-Q|>D$ is satisfied, where $D$ is a fixed threshold distance, and consequently the pair must no longer be considered.
The calculation of $r_{\max }$ and $r_{\min }$ has computational complexity of order $N$. Therefore, the first filter has a computational complexity of order $N^{2}$ because we compare all possible pairs, but each comparison is so fast that there is no problem even if $N$ is extremely large.

\subsection{Filter II}

Filter II, proposed in this work, is an improvement of the second filter formulated by Hoots et al. (1984). They developed a geometrical filter based on the relative geometry between the orbit of the primary object and the orbit of the secondary. The main idea of this filter is that, given a pair of orbits, the orbital distance, denoted by $d_{\text {min }}$, is the minimum value of the distance between all possible positions assumed by two objects moving along two elliptical orbits. The minimum distance is not necessary along the nodal line of intersection between the two orbital planes. If $d_{\min }$ is always greater than a threshold distance $D$, then the pair of objects cannot have a close encounter of interest.

The iterative method proposed by Hoots et al. (1984) to find the minimal points, which are the orbital points where the minimum distances are reached, takes as initial conditions two points close to the mutual nodes or in their neighbourhood. Although this is true for objects whose mutual inclination is not small, it has been shown that the minimum points can be far away from the mutual nodes (Gronchi 2002). This is one of the reasons why we should improve the second filter of Hoots et al. (1984). Another reason is that we can apply novel techniques to compute the orbital minimum distance.

The distance between two Keplerian orbits is commonly used in the literature about asteroids to discover whether two objects moving along these orbits can undergo a very close approach or, indeed, a collision. If the distance is large enough, then there is no possibility of such an event. However, two confocal Keplerian orbits might become close at another pairs of points. Thus, it is necessary to compute not only the absolute minimum (i.e. the MOID) but also all its local minimal distance values.

The second filter proposed here is based on the definition of the local minimal distances introduced by Gronchi \& Tommei (2007). By simply changing the sign of the distance maps in suitable subset of their domain, we obtain the two-orbit configuration spaces, more regular maps, called distances with sign. For each pair of objects, it is possible to compute their signed distances at any time in the interpolation process. If there is a change of sign in one of the local minimal distances (i.e. an orbit crossing occurs), the pair passes the second filter; otherwise, the pair is excluded for a possible collision.

Let us explain in detail the second filter. We consider a set $\mathcal{E}=\left(\mathcal{E}_{s_{1}}, \mathcal{E}_{s_{2}}\right)$ of 10 elements, composed by two subsets of five elements each, such that $\mathcal{E}_{s_{j}}$ defines the geometric configuration of the $s_{j}$ th orbit $(j=1,2)$. Furthermore, we consider a vector $V=\left(v_{s_{1}}, v_{s_{2}}\right)$, such that $v_{s_{j}}$ defines a parameter along the $s_{j}$ th orbit $(j=1,2)$. Because we are working with equinoctial elements, we can choose $\mathcal{E}_{s_{j}}=\left(a_{s_{j}}, h_{s_{j}}, k_{s_{j}}, p_{s_{j}}, q_{s_{j}}\right)$ for the orbit configuration $(j=1,2)$, and $V=\left(\lambda_{s_{1}}, \lambda_{s_{2}}\right)$ as the mean longitude vector. In an inertial reference frame, with the origin at the common focus of the objects, we denote by $\mathcal{X}_{s_{j}}=\mathcal{X}_{s_{j}}\left(\mathcal{E}_{s_{j}}, v_{s_{j}}\right)$ the Cartesian coordinates of two bodies $(j=1,2)$. Then, for each two-orbit configuration $\mathcal{E}$, the Keplerian distance function $d$ is defined as the map:

$$
\begin{aligned}
d(\mathcal{E},-): \mathcal{V} & \rightarrow \mathbb{R}^{+} \\
V & \mapsto d(\mathcal{E}, V)=\left|\mathcal{X}_{s_{1}}-\mathcal{X}_{s_{2}}\right|
\end{aligned}
$$


Here, $|\cdot|$ is the Euclidean norm in $\mathbb{R}^{3}$ and $\mathcal{V}=\mathbb{T}^{2}$ if both orbits are bounded, $\mathcal{V}=S^{1} \times \mathbb{R}$ if only one is bounded, and $\mathcal{V}=\mathbb{R} \times \mathbb{R}$ if they are both unbounded. In our work, we only consider elliptical orbits, so $\mathcal{V}$ is the two-dimensional torus $\mathbb{T}^{2}$.

The critical points of the function $d$ are computed through those of $d^{2}$ to avoid problems of differentiability when it vanishes. They can be efficiently computed following the computational methods developed by Gronchi (2005). The minimal points correspond to the possible close approaches of the two objects. Numerical computations show that the number of minimal points can be up to four when the trajectories are non-overlapping conics or two coplanar circles (Gronchi 2002). Furthermore, if we exclude these exceptional cases, the number of crossing points are at most two (Gronchi \& Tommei 2007).

Generically, there is a finite number of critical points. Let $V_{h}(\mathcal{E})=$ $\left[v_{1}^{h}(\mathcal{E}), v_{2}^{h}(\mathcal{E})\right]$ be the $h$ th local minimal point of the function $d^{2}$. Let

$\mathcal{X}_{1}^{h}(\mathcal{E})=\mathcal{X}_{1}\left[\mathcal{E}_{1}, v_{1}^{h}(\mathcal{E})\right], \quad \mathcal{X}_{2}^{h}(\mathcal{E})=\mathcal{X}_{2}\left[\mathcal{E}_{2}, v_{2}^{h}(\mathcal{E})\right]$

be the Cartesian coordinates corresponding to the local minimal points of index $h$. For every two-orbit configuration $\mathcal{E}$, the local minimal distance is the map defined as

$d_{h}(\mathcal{E})=d\left[\mathcal{E}, V_{h}(\mathcal{E})\right]=\left|\mathcal{X}_{1}^{h}(\mathcal{E})-\mathcal{X}_{2}^{h}(\mathcal{E})\right|$,

which represents the distance between the two points corresponding to the $h$ th local minimal point.

The orbit distance corresponds to the absolute minimum of the function $d(\mathcal{E},-)$, and, for every $\mathcal{E}$, it is defined by the map:

$d_{\min }(\mathcal{E})=\min _{h}\left\{d_{h}(\mathcal{E})\right\}$.

The functions $d_{h}$ and $d_{\min }$ present three main problems (Gronchi $\&$ Tommei 2007). First, they are singular at orbit crossing (i.e. their derivatives with respect to the orbital elements are not defined when they vanish). Secondly, the function $d_{h}$ might become ambiguous after a bifurcation at a critical point. In particular, it is not guaranteed that a minimum point does not change its type, and becomes, for instance, a maximum point, after a bifurcation. Thirdly, the function $d_{\text {min }}$ might lose its regularity when there is an orbit configuration $\mathcal{E}$ such that, in a neighbourhood of $\mathcal{E}$, we can find two local minimal distance maps that exchange their role as absolute minimum. In this case, $d_{\min }$ can lose its regularity even without vanishing (without orbit crossing).

To overcome the singularity when the distances $d_{h}, d_{\min }$ vanish, Gronchi \& Tommei (2007) have proposed a method to define a map of new orbital distances by simply changing their sign on a suitable subset of the domain. The 'distances with sign' are regular in the neighbourhood of most crossing configurations. The method is quite complicated but it turns out to have a simple geometric interpretation.

For a given two-orbit configuration $\mathcal{E}$, let $\tau_{1}^{h}, \tau_{2}^{h}$ be the tangent vectors to the orbits at their local minimal points $V_{h}$ and let $\tau_{3}^{h}$ be the cross product of $\tau_{1}^{h}$ and $\tau_{2}^{h}$. Let $\Delta_{h}=\mathcal{X}_{2}^{h}-\mathcal{X}_{1}^{h}$ be the line joining the points corresponding to the local minimal points. Then, the local orbit distance with a sign of index $h$ is given by

$\tilde{d}_{h}=\left(\hat{\tau}_{3}^{h} \cdot \hat{\Delta}_{h}\right) d_{h}$,

where $\hat{\tau}_{3}^{h}$ and $\hat{\Delta}_{h}$ are the corresponding unit vectors. Indeed, $\tau_{3}^{h}$ and $\Delta_{h}$ are parallel and the sign of $\tilde{d}_{h}$ is chosen according to the orientation of $\Delta_{h}$ (Fig. 1). The minimum distance with sign $\tilde{d}_{\text {min }}$ is defined in a similar way. More details about the regularization method can be found in Gronchi \& Tommei (2007).

The new distance maps $\tilde{d}_{h}$ and $\tilde{d}_{\text {min }}$ are generically not singular at orbit crossing. In this way, we solve the first problem that the non-

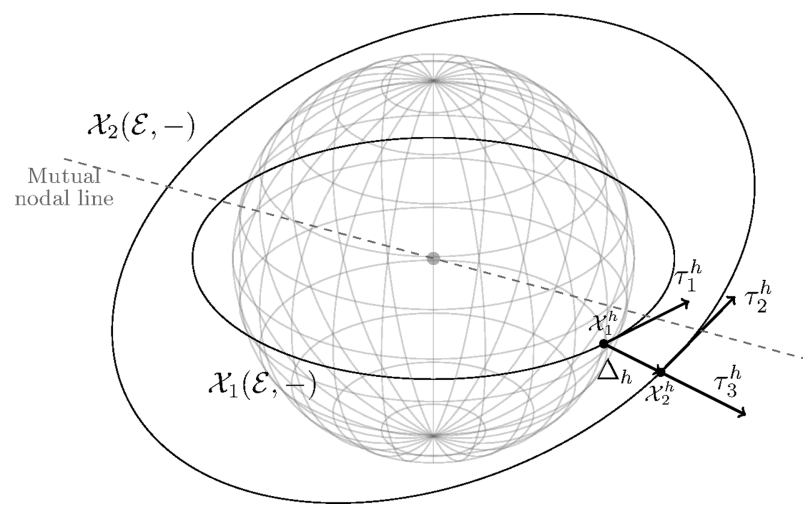

Figure 1. Geometric interpretation of $\tilde{d}_{h}$.

regularized functions present. However, $\tilde{d}_{h}$ and $\tilde{d}_{\min }$ are not defined, with or without orbit crossings, at the two-orbit configurations, such that $\tau_{1}^{h}$ and $\tau_{2}^{h}$ are parallels (tangent configurations).

The functions $\tilde{d}_{h}$ might still lose their regularity when a bifurcation occurs. This problem can be overcome by restricting the domain in the neighbourhood of a non-degenerate two-orbit configuration. A configuration $\mathcal{E}$ is non-degenerate if all the critical points of the Keplerian distance function are non-degenerate, that is following the condition

$\operatorname{det} \mathcal{H}_{V}\left(d^{2}\right)\left[\mathcal{E}, V_{h}(\mathcal{E})\right] \neq 0$,

where $\mathcal{H}_{V}\left(d^{2}\right)$ is the Hessian matrix of $d^{2}$. If the configuration $\mathcal{E}^{*}$ is non-degenerate, then there exists a neighbourhood $\mathcal{W}$ of $\mathcal{E}^{*} \in \mathbb{R}^{10}$ such that no bifurcation occurs and the number of critical points does not change for every $\mathcal{E} \in \mathcal{W}$, and each $\mathcal{E}$ has the same type of critical points.

The function $\tilde{d}_{\min }$, as the function $d_{\min }$, might lose its regularity when there is an orbit configuration $\mathcal{E}$ in which two distinct local distances (e.g. $\left.\tilde{d}_{1}, \tilde{d}_{2}\right)$ reach the same value, $\tilde{d}_{1}(\mathcal{E})=\tilde{d}_{2}(\mathcal{E})$, which corresponds to $\tilde{d}_{\min }(\mathcal{E})$. Then, in the neighbourhood of $\mathcal{E}$, we can have two local minima that exchange their role as absolute minimum, and $\tilde{d}_{\min }$ can lose its regularity even without vanishing (without orbit crossing). To overcome this problem, we control the evolution of the two smallest distance functions, denoted by $\tilde{d}_{1}$ and $\tilde{d}_{2}$.

We can now explain how filter II works. Given a pair of orbits, if no bifurcation and no tangent configuration occur, we compute, for each instant of time $t_{1} \leq t \leq t_{m}$, where $m$ is the number of time nodes, the functions $\tilde{d}_{1}(t)$ and $\tilde{d}_{2}(t)$. We only care about these two functions because the number of crossing points are, at most, two, as already mentioned. If there is a change of sign at any instant of time in any of the functions $\tilde{d}_{1}(t)$ or $\tilde{d}_{2}(t)$, it means that an orbit crossing is possible, and consequently the pair must be considered for the next filter. If neither of the distances change their sign, then there is no orbit crossing and the pair can be disregarded.

\subsection{Filter III}

Filter III is the time filter, and it is applied to the pairs that have passed both filter I and filter II, which means that there is an orbit crossing between the two orbits. Given an index pair $(r, s)$ that passes filters I and II, we have the configuration $\mathcal{E}=\left(\mathcal{E}_{r}, \mathcal{E}_{s}\right)$ and we can compute for each time $t_{1} \leq t \leq t_{m}$ the position of the piece of 
space debris along the orbit. The distance between the two objects is simply given by

$d[\mathcal{E}(t), V(t)]=\left|\mathcal{X}_{r}-\mathcal{X}_{s}\right|$,

where $\mathcal{X}_{r}$ and $\mathcal{X}_{s}$ are the Cartesian coordinates of the two bodies at time $t$. If the distance is greater than a threshold distance $D$, the collision can be excluded at time $t$. This procedure is iterated for any time. The pair can be excluded if there is never a time when the distance between the objects is less than $D$. Otherwise, the pair of objects is at real risk of collision.

\section{RESULTS}

We apply the filter sequence to a population of 864 pieces of space debris to obtain the pairs of orbits that are at real risk of collision. Then, we compare filter I described in this paper with the filer proposed by Hoots et al. (1984). We also analyse the evolution of the distance function $\tilde{d}_{1}$ of a local minimum point to show the way an orbit crossing is found. Finally, we present the dependency of the filter sequence with respect to the time-step considered.

\subsection{Application of the filter sequence}

In this paper, we consider 864 objects, which translates into 372816 pairs to be considered for collisions. The orbit evolution of these objects is computed numerically by a symplectic integration scheme (Hubaux et al. 2012), considering the Earth's gravitational potential, luni-solar and planetary gravitational perturbations and direct solar radiation pressure. In particular, we propagate each object over a period of $10 \mathrm{~d}$ with a time-step of $36 \mathrm{~min}$ (400 time nodes), which represent the ephemerides of the entire population. The threshold distance considered is $100 \mathrm{~m}$. In our research, we consider a timestep of $0.05 \mathrm{~d}(\approx 1.2 \mathrm{~h})$, and consequently the number of time nodes we interpolate is around 200.

We apply filter I to the entire population of 864 objects. There are 218459 pairs that pass this filter, which will consequently be analysed with the second filter. Note that filter I excludes 154357 pairs, which means that 41.4 per cent of the pairs of objects have an orbit configuration without risk of collision.

Filter II is applied to the 218459 pairs that passed filter I. We find that only 49739 pairs pass filter II, and these will be analysed with the third filter. In this case, filter II excludes 168720 pairs, which represents 77.2 percent of the pairs considered using the second filter. There are 323077 pairs excluded after applying filters I and II, which represents 86.7 per cent of all possible pairs.

Finally, we apply the third filter, or time filter, to the 49739 pairs that have passed filters I and II, which are consequently at tentative risk of collision. Filter III excludes 49739 pairs, which represents 100 percent of the pairs considered, meaning that there is no risk of collision for any pair of objects.

In conclusion, the filter sequence excludes 372816 pairs of objects, which represents 100 percent of the entire population. This percentage allows us to confirm the powerful and efficiency of this filter sequence to determine pairs of objects at real risk of collision.

\subsection{Comparison between our filter I and that of Hoots et al. (1984)}

If we use the first filter proposed by Hoots et al. (1984), which consists of a simple comparison of the perigees and apogees of each pair of orbits, we observe that 240189 pairs pass the filter, which means that 132627 pairs have been excluded. This represents 35.6 per cent

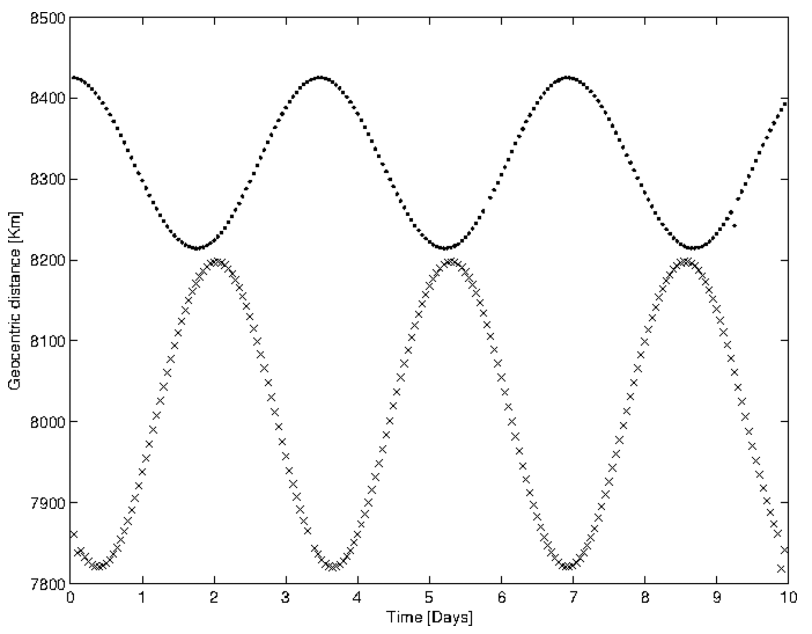

Figure 2. Geocentric distances of a pair of orbits in the catalogue (denoted by crosses and dots) as a function of time. There is no orbit crossing.

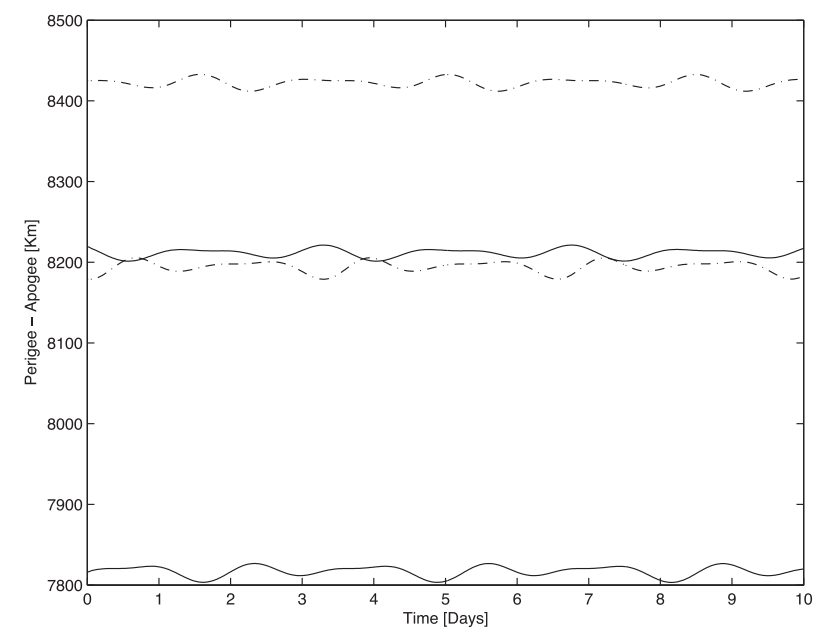

Figure 3. Evolution of the perigee (dashed line) and apogee (dash-dotted line) for a given couple of orbits as a function of time. There is an orbit crossing within $1 \mathrm{~d}$ of evolution.

of the entire population. Consequently, our filter I detects 21730 false positives, which passed the first filter proposed by Hoots et al. (1984). Note that the main problem of the first filter presented by Hoots et al. (1984) was computing the ephemerides using the osculating elements. This comparison has been done with our powerful interpolation ephemerides table instead of the osculating elements, even though our proposed filter I improves the filter of Hoots et al. (1984)

For clarity, Fig. 2 illustrates the geocentric distance of two objects, while Fig. 3 illustrates the evolution of the perigee and apogee of the same objects. In this particular case, we observe a false positive in the perigee-apogee criteria because, if we consider the geocentric distance of the pair of orbits, there is no risk approach, while if we consider the perigee-apogee criteria there is the possibility of an orbit crossing. Using these figures, we illustrate the efficiency of our filter I against that of Hoots et al. (1984).

\subsection{Evolution of the distance function in filter II}

Filter II uses the signed distance functions $\tilde{d}_{1}$ and $\tilde{d}_{2}$ presented in equation (11), which represent the distances between two local 


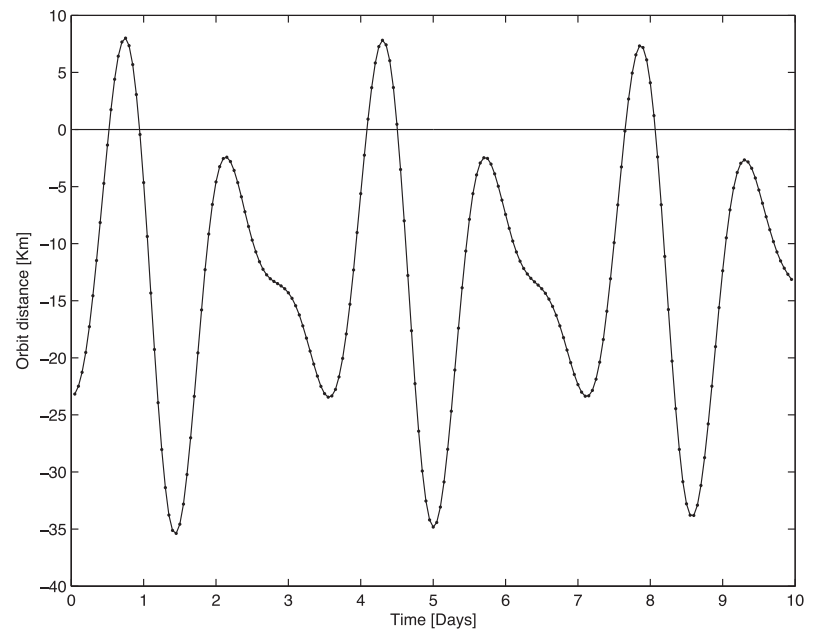

Figure 4. Evolution of the function $\tilde{d}_{1}$ with a change of sign in the function, which implies that there is an orbit crossing.

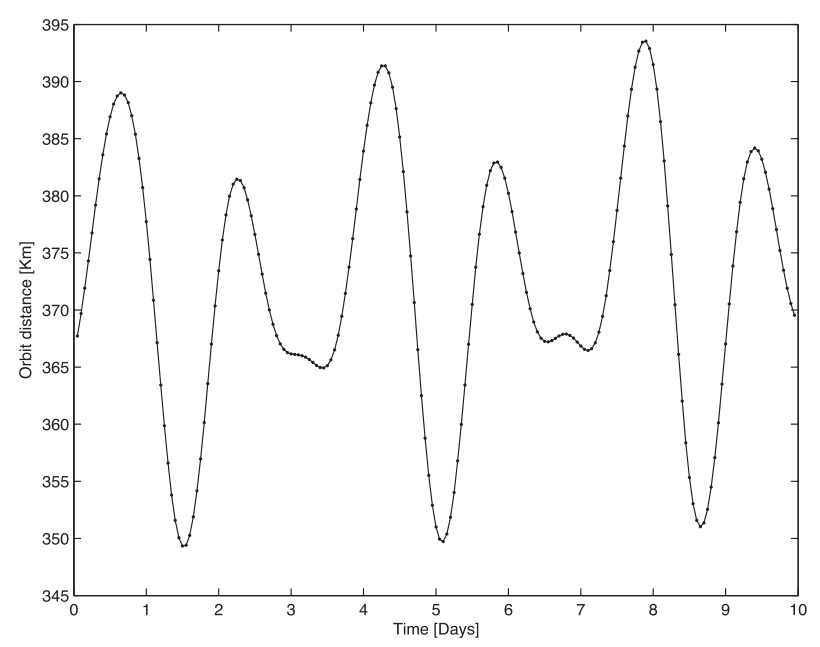

Figure 5. Evolution of the function $\tilde{d}_{1}$ without a change of sign in the function, which implies that there is no orbit crossing.

minimal points. If one of the functions presents a change of sign, there is an orbit crossing; otherwise, there is not. Fig. 4 illustrates the evolution of the function $\tilde{d}_{1}$ when an orbit crossing occurs, and consequently when a change of sign in the function is present. In this case, the first orbit crossing occurs within less than $1 \mathrm{~d}$ of propagation. Fig. 5 illustrates the evolution of the function $\tilde{d}_{1}$ in a pair of orbits where there is no orbit crossing, and consequently there is no change of sign in the function $\tilde{d}_{1}$.

\subsection{Dependence of filter sequence on time-step}

In this section, we apply the filter sequence to the same population of 864 pieces of space debris, but in this case, we consider a timestep of $0.008 \mathrm{~d}(\approx 12 \mathrm{~min})$. The number of time nodes is now around 1300 .

Table 1 shows the number of pairs that pass the subsequent filters if we apply the filter sequence to the same population with different time-steps. We observe that the smaller the time-step is, the more accurate the results are, and consequently we can exclude more pairs at risk of collision. Note that when the time-step is 0.05 , we obtain
Table 1. Pairs to be considered after applying each filter to a population of 864 pieces of space debris. Two different time-steps are used, and consequently the number of pairs that pass each filter changes.

\begin{tabular}{lcllll}
\hline \multicolumn{3}{c}{$d t=0.05$} & & \multicolumn{3}{c}{$d t=0.008$} \\
\hline Filter I & Filter II & Filer III & Filter I & Filer II & Filer III \\
218459 & 49739 & 0 & 216837 & 49739 & 0 \\
\hline
\end{tabular}

about 1622 false positives when applying filter I, which means that they are considered at real risk of collision, although they are not, because if we reduce the time-step, they are excluded. In the second filter, there are 256 false positives, and in the third filter, there are no false positives, because in both cases the number of pairs at real risk of collision is zero.

As can be seen, the more precision we need, the smaller the timestep needs to be. However, the smaller the time-step is, the more computational time is required. If we need a first approach, with certain feasibility, the time-step can be the biggest one. Thus, the computational time will be substantially reduced.

\section{ORBIT UNCERTAINTY}

The only limitation of the filter sequence is the poor orbit determination used to compute the ephemerides table. This poor orbit determination is a result of unannounced manoeuvres or a large effect of drag perturbation. However, the uncertainty, so far not taken into account in this work, is also a problem. The uncertainty is the error produced when computing the locations of pieces of space debris in their orbits because of different observational errors. This means that each piece of space debris is associated with a covariance matrix indicating the quality of the computed position.

If we include the uncertainty of each object at each time in the ephemerides table, which is the probability of being exactly in that point at a given instant of time, we must use it in the filter sequence. Filter I can be improved by including the uncertainty in the calculation of the minimum and maximum distances, and then the method will be more realistic. Filter II can be improved by including the computation of the uncertainty on the signed distances, due to the uncertainty on the orbits. The property of differentiability of these functions at orbit crossing is now fundamental if one wants to apply a covariance propagation formula, requiring regularity, to compute a meaningful uncertainty for the distances.

\section{CONCLUSIONS AND FUTURE WORK}

In this paper, we have focused on the avoidance of space debris collisions. To that end, we have designed a filter sequence that is able to disregard pairs of orbits that are not at real risk of collision. In detail, we have based the filter sequence on two procedures. The first procedure is the storage of the orbital ephemerides in a similar way as the SPICE kernels used by NASA, which allows easy access to the data with a low RAM memory. The second procedure concerns three filters based on the mutual geometry of all pairs of orbits considered, and the time coincidence between them. We have applied this filter sequence to a population of 864 pieces of space debris, and we have compared our filter I with the one presented by Hoots et al. (1984) to prove the efficiency of our methodology. We have also analysed the influence of the time-step applied in the filter sequence, and we have discussed the results obtained.

As future work, we aim to consider some improvements of the filter sequence. First, there are cases in which the computation 
of the minimum distance fails, coinciding with cases where the tangent vectors are parallel. Secondly, the time required is too long, and consequently some parallelization techniques can be applied. Finally, the ephemerides table can be computed more precisely (e.g. by including the atmospheric drag).

There are two different ways to continue this work. The first way is to consider more than 864 pieces of space debris (e.g. the approximately 21000 objects that comprise the TLE catalogue), with their uncertainties, and to include the uncertainty in the functions of the filter sequence in order to compute pairs of objects at real risk of collision. The second way is to consider the TLE catalogue without orbit uncertainty, and to use the filter sequence proposed in this work for the entire catalogue of space debris. The increase of the computational cost can be reduced by applying some parallelization techniques.

\section{ACKNOWLEDGEMENTS}

This paper presents the research results of the Belgian Network Dynamical Systems, Control, and Optimization (DYSCO), funded by the Interuniversity Attraction Poles Programme, initiated by the Belgian State, Science Policy Office. The scientific responsibility rests with its author(s). The authors would especially like to thank Giovanni F. Gronchi, who agreed to review this work.

\section{REFERENCES}

Acton C. H., 1996, Planet Space Sci., 44, 65

Broucke R. A., Cefola P. J., 1972, Celestial Mech., 5, 303

Casanova D., Lemaître A., 2014, Celest. Mech. Dyn. Astron., submitted
Delsate N., Lemaître A., Carletti T., Robutel P., 2010, Celest. Mech. Dyn. Astron., 108, 275

Dimare L., Farnocchia D., Gronchi G. F., Milani A., Bernardi F., Rossi A., 2011, in Ryan S., ed., Advanced Maui Optical and Space Surveillance Technologies Conference. Maui Economic Development Board, Maui, E51

Gronchi G. F., 2002, SIAM Journal on Scientific Computing, 24, 61

Gronchi G. F., 2005, Celest. Mech. Dyn. Astron., 93, 295

Gronchi G., Tommei G., 2007, Discrete and Continuous Dynamical Systems, Series B, 7, 755

Gronchi G. F., Tommei G., Milani A., 2006, in Valsecchi G. B., Vokrouhlický D., Milani A., eds, Proc. IAU Symp. 236, Near Earth Objects, Our Celestial Neighbors: Opportunity and Risk. Cambridge Univ. Press, Cambridge, p. 3

Gronchi G. F., Dimare L., Milani A., 2010, Celest. Mech. Dyn. Astron., 107, 299

Hoots F. R., Crawford L. L., Roehrich R. L., 1984, Celest. Mech., 33, 143

Hubaux C., Lemaître A., Delsate N., Carletti T., 2012, Adv. Space Res., 49, 1472

Lemaître A., Delsate N., Valk S., 2009, Celest. Mech. Dyn. Astron., 104, 383

Milani A., Tommei G., Chesley S., Sansaturio M., Valsecchi G., 2005, Icarus, 173,362

Pardini C., Anselmo L., 2011, Adv. Space Res., 48, 557

Valk S., Lemaître A., Anselmo L., 2008, Adv. Space Res., 41, 1077

Valk S., Lemaître A., Deleflie F., 2009a, Adv. Space Res., 43, 1070

Valk S., Delsate N., Lemaître A., Carletti T., 2009b, Adv. Space Res., 43, 1509

Woodburn J., Coppola V., Stoner F., 2010, Adv. Astronaut. Sci., 135, 1157

This paper has been typeset from a $\mathrm{T}_{\mathrm{E}} \mathrm{X} / \mathrm{LT}_{\mathrm{E}} \mathrm{X}$ file prepared by the author. 